\title{
Komplementärmedizin auf dem Prüfstand
}

\begin{abstract}
Das EMR registriert im Auftrag vieler Krankenkassen komplementärmedizinisch tätige Therapeuten in der Schweiz. Nach dreijähriger Tätigkeit konnte das EMR bereits 12000 Anträge prüfen; davon wurden rund 1000 abgelehnt. Die Leiterin des EMR, Frau Dr. med. Silva Keberle, gibt uns einen Einblick ihre ebenso faszinierende wie kräftezehrende Tätigkeit.
\end{abstract}

Was macht das EMR?

Das ErfahrungsMedizinische Register (EMR) ist eine Abteilung der Firma Eskamed AG und besteht seit 1995. Bis Ende 1998 hat das EMR ausschliesslich Registrierungen von komplementärmedizinisch tätigen Therapeutinnen und Therapeuten im Auftrag der Swica durchgeführt. Anfang 1999 wurde eine Vereinbarung mit der IGQSK (Interessengemeinschaft für die Qualitätssicherung in der Komplementärmedizin) abgeschlossen. Zur IGQSK gehören die Versicherer Helsana, CSS Versicherung, Swica, Concordia und Wincare. Die Vereinbarung beinhaltet, dass das EMR bei den interessierten Therapeutinnen und Therapeuten prüft, ob sie die Anerkennungskriterien erfüllen. Jeder Therapeut erhält eine Konkordatsnummer zugeteilt; alle zwei Wochen werden die aktuellen Listen den Versicherern zur Verfügung gestellt. Inzwischen haben sich über 40 Krankenversicherer dem EMR angeschlossen.

\section{Wozu braucht es eine solche Registrierung überhaupt? \\ Mit den dringlichen Bundesbeschlüssen bei der} Einführung des neuen KVG wurden Versicherungsleistungen im Bereich der Komplementärmedizin, die bis anhin über die Grundversicherung abgerechnet werden konnten, alle in den Bereich der Zusatzversicherungen verlegt. Davon ausgenommen sind die fünf Methoden, die gemäss KVG über die Grundversicherung abgerechnet werden können, sofern die Leistung von einem hierfür zugelassenen Arzt erbracht wird. Zum Schutz der Patienten ist es aber wichtig, dass die Qualität der Leistungserbringer in der Komplementärmedizin auch im Bereich der Privatversicherung soweit wie möglich definiert und verifiziert werden kann. Dies bedeutet, dass diese Therapeuten über eine gute Ausbildung verfügen sollten, und dass sie diese Qualität mit Hilfe einer regelmässigen Fortbildung erhalten müssen. In der Schweiz ist praktisch alles reglementiert, nicht aber die Ausbildung von nicht-ärztlichen Komplementärtherapeuten, die doch immerhin kranke Menschen behandeln.
Das EMR in Kürze

Das EMR hat die Aufgabe, im Auftrag der angeschlossenen Krankenversicherer zu prüfen, ob die Therapeuten, die sich beim EMR registrieren lassen wollen, eine ausreichende Ausbildung in den angemeldeten Therapiemethoden abgeschlossen haben.

- Es werden ausschliesslich die Ausbildungsund Fortbildungsdokumente überprüft, und es wird kontrolliert, ob die weiteren geforderten Anerkennungskriterien erfüllt sind.

- Für alle Therapeuten, die sich beim EMR registrieren lassen, gelten dieselben Mindestkriterien bezüglich Aus- und Fortbildung.

- Das EMR leistet als anerkannte, unabhängige Kontrollstelle einen Beitrag zur Qualitätssicherung auf dem Gebiet der Komplementärmedizin. Dabei stützt sich das EMR auf die Zusammenarbeit mit Sachverständigen der Versicherer, mit Vertretern von Schulen und Verbänden und mit dem Fachbeirat.

- Qualitätssicherung in der Komplementärmedizin ist eine komplexe Aufgabe. Die von den interessierten Krankenversicherern initiierte zentrale Registrierung durch das EMR ist ein erster Schritt einer langfristigen Entwicklung. Das Ziel ist eine systematische und transparente Qualitätssicherung.

Das heisst, EMR registriert nicht nur, sondern kontrolliert auch?

Das EMR kontrolliert, ob eine ausreichende Aus- und Fortbildung in den angemeldeten Therapiemethoden dokumentiert werden kann. Das EMR beurteilt die Therapeuten nicht bezüglich ihrer individuellen Qualität und führt auch keine Prüfungen durch; das ist - ähnlich wie bei der FMH - Sache der Fachverbände und Ausbildungsstätten. Für alle Therapeuten, die sich beim EMR registrieren lassen, gelten bei derselben Therapiemethode dieselben Mindestkriterien bezüglich Aus- und Fortbildung. Es besteht also - im Gegensatz zu früher - volle Transparenz. Die geforderten Kriterien können von jedermann unter www.emr.ch eingesehen werden. Auch muss sich der einzelne Therapeut nicht mehr bei jeder Versicherung anmelden, erst noch nicht wissend, welches die Anforderungen dort sind. Das EMR bringt also den Therapeuten auch eine deutliche Verbesserung bezüglich administrativer Verfahren.

Wie wurden diese Therapeuten früher anerkannt, als es den EMR noch nicht gab?

Die meisten Versicherer haben sogenannte Therapeutenlisten geführt. Zweck dieser Listen war einerseits die Erfassung der Personalien und andererseits eine mehr oder weniger detaillierte Qualitätskontrolle. Bei 


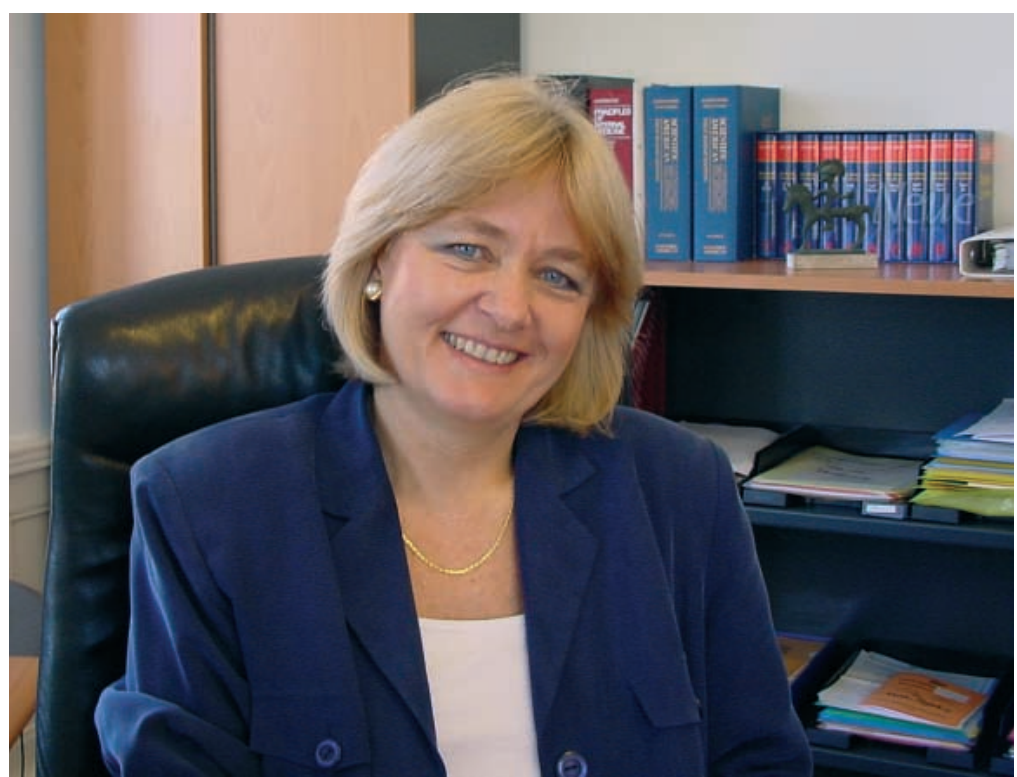

Frau Dr. med. Silva Keberle beschäftigt sich seit Jahren mit Fragen der Qualitätssicherung in der Schul- und Komplementärmedizin. Silva Keberle ist Inhaberin der Firma Eskamed AG, das EMR ist eine Abteilung der Eskamed AG. Die Eskamed AG ist eine Agentur, die Projekte im Gesundheitswesen entwickelt und umsetzt.

mindestens 300 Komplementärmethoden, die auf dem Schweizer Markt angeboten werden, und bei geschätzten 20000 Komplementärtherapeuten, die solche Methoden einzeln oder in Kombination anbieten, wurde die angestrebte Qualitätskontrolle für die jeweiligen Sachbearbeiterinnen der Versicherer jedoch zunehmend belastend. Erschwerend kam hinzu, dass es kaum brauchbare Dokumentationen zu den verschiedenen Methoden gab, ebensowenig wie ein Verzeichnis der Fachverbände und Schulen der Komplementärmedizin in der Schweiz. Ende 1998 umfasste die grösste Therapeutenliste bei den Versicherern über 13000 Therapeuten.

\section{Woher hat das EMR die Kompetenz für diese Aufgabe?}

Es gibt in der ganzen Schweiz wohl keine einzelne Person oder einzelne Institution, die zu den rund 200 Methoden, welche die Versicherer anerkennen, ein umfassendes Fachwissen aufweist. Darum hat das EMR seine Kompetenz modular und vernetzt aufgebaut. Zum einen betreut das EMR bereits seit $1995 \mathrm{im}$ Mandatsverhältnis die Therapeutenliste für die Swica und hat in dieser Zeit gegen 3000 Bewerbungen bearbeitet und dadurch viele Erfahrungen sammeln können. Das EMR wird zudem von drei hoch kompetenten Fachgremien unterstützt und auch kontrolliert. Es sind dies die Task Force, die sich aus Experten der Versicherer sowie erfahrenen Vertretern der Komplementärmedizin zusammensetzt, der «Runde Tisch" mit ebenfalls ausgewiesenen Therapeutinnen und Therapeuten der Komplementärmedizin und der 14köpfige Fachbeirat unter dem Präsidium von Professor Reinhard Saller. Bei allen Mitgliedern dieser Gremien handelt es sich um Personen, die eine grosse Erfahrung in der Komplementärmedizin aufweisen.
Wenn ich als Therapeut ein komplementärmedizinisches Verfahren anbieten und verrechnen lassen möchte, muss ich mich im EMR eintragen lassen?

Nur dann, wenn Ihr Patient bei einem Versicherer die entsprechende Police hat und eine Rückerstattung wünscht. Viele Therapeuten sind nicht beim EMR registriert und haben dennoch eine volle Sprechstunde. Dazu kommt, das nicht wenig Versicherer zusätzlich zur EMR-Liste gewisse Therapeuten direkt anerkennen, so zum Beispiel zahlt die Swica Leistungen von kantonal anerkannten Therapeuten, auch wenn sie nicht beim EMR registriert sind.

\section{Gibt es auch andere Anbieter von solchen} Registrierungen?

$\mathrm{Ja}$, es gibt zum Beispiel die ASCA, die vor allem in der Romandie stark vertreten ist. Und dann haben viele der Versicherer, die sich dem EMR nicht angeschlossen haben, so zum Beispiel die Visana, die Groupe Mutuel oder die EGK, ein eigenes, differenziertes Anerkennungsverfahren.

\section{Warum machen nicht der Bund oder die Kantone} diese Qualitätskontrolle?

An sich wäre dies begrüssenswert. Bei der kantonalen Zulassung ist das ja auch schon teilweise der Fall. Bei der Vereinbarung zwischen den Komplementärtherapeuten und den Versicherern geht es jedoch um einen privatrechtlichen Vertrag, bei dem die Krankenversicherer ihre Kriterien definieren und die Therapeuten entscheiden können, ob sie auf diese Kriterien eingehen wollen oder nicht. Auch wird bei den Kantonen die Qualitätskontrolle bei Komplementärtherapeuten sehr unterschiedlich gehandhabt. In vielen Kantonen ist das Praktizieren einer Komplementärmethode verboten, zum Beispiel bisher in Zürich. In den permissiven Kantonen variiert das Anerkennungsverfahren. So kennt Basel-Landschaft eine strenge Prüfung für wenige ausgewählte Methoden. In einigen Kantonen wird eine Prüfung für Naturheilpraktiker abgenommen, bei der die später praktizierten Methoden jedoch nicht oder eher allgemein abgefragt werden. Wieder andere Kantone sind bei der Zulassung sehr liberal. Der Begriff «kantonal approbiert» lässt also keinen einheitlichen Schluss über die Qualität der Ausbildung zu. Insbesondere ist bei diesen Therapeuten oft nicht belegbar, dass sie die Methode, die sie bei Patienten einsetzen, wirklich erlernt haben. Eine kantonale Zulassung wird vom EMR jedoch immer positiv in die Beurteilung eines Antrags einbezogen.

Welches sind die wichtigsten Anerkennungskriterien beim EMR?

$\mathrm{Zu}$ den wichtigsten Qualitätskriterien für die Registrierung beim EMR gehören der Nachweis eines schulmedizinischen Basiswissens, das in der Ausbildung von den entsprechenden Schulen vermittelt wird. Konkret heisst das, dass - je nach Methode mehrere hundert Stunden schulmedizinischer Unterricht nachgewiesen werden müssen. Dazu kommt 
natürlich die Dokumentation einer abgeschlossenen methodenspezifischen Ausbildung, die Bestätigung der Berufserfahrung (250 Patientenstunden), die limitierte Anmeldung für maximal drei Methoden, das Minimum von mindestens 20 Prozent beruflicher Tätigkeit pro Methode, der Abschluss einer Berufshaftpflichtversicherung, die Dokumentationspflicht, also das Führen einer Krankengeschichte und natürlich die Verpflichtung zur jährlichen Fortbildung. Diese Kriterien scheinen vielleicht eher banal, aber unsere Erfahrungen zeigen, dass die Erfüllung dieser Kriterien bisher keineswegs selbstverständlich war.

Wie geht das EMR mit der Besitzstandswahrung um? Die schon seit langem tätigen Therapeuten konnten ja früher nicht wissen, wie die EMRKriterien dereinst sein würden.

Auch hier haben wir uns an das Konzept der FMH mit den Fähigkeitsausweisen angelehnt. Es geht um das wohlerworbene Recht. Zum einen garantieren in solchen Fällen die entsprechend qualifizierten Fachverbände, dass ihre "altgedienten" Mitglieder den Qualitätskriterien des EMR entsprechen. Und dann wird die Berufserfahrung langjährig tätiger Therapeuten mit einem Berechnungsschlüssel in die Beurteilung einbezogen. Genügt das nicht, so kann die Task force oder der Fachbeirat aufgrund der individuellen Situation eine Anerkennung aussprechen, auch wenn die geforderte Ausbildung nicht lückenlos belegt werden kann. Es sind also verhältnismässig wenige Therapeuten, welche die EMR-Hürde nicht schaffen. Vor dem EMR waren von 13000 Therapeuten auf der grössten Versichererliste etwa 3000 abgelehnt. Beim EMR sind es, nach drei Jahren, erst rund 1000 bei inzwischen bald 12000 geprüften Anträgen.

Ist das EMR also eher ein wohlwollender Türöffner als ein strenger Gatekeeper?

Ich würde sagen, ein wohlwollender Gatekeeper.

\section{Welche Methoden können beim EMR registriert werden?}

Rund 200 Methoden, sie können unter www.emr.ch eingesehen werden. Diese Methoden bilden den gemeinsamen Nenner derjenigen Methoden, welche die einzelnen Versicherer zu Zeiten vor dem EMR bereits anerkannt haben. Es war für uns ein beachtlicher Aufwand herauszufinden, welche Philosophie und welches Angebot den vielen weniger bekannten Methoden genau zugrunde liegt. Auch hier gibt es bisher keinen Kataster. Wir haben aber in der Zwischenzeit alle 200 Methoden systematisch beschrieben und verfügen somit über eine einzigartige Dokumentation. Anekdotisch kann ich erwähnen, dass wir anfangs zum Beispiel nicht wussten, um was es sich bei der Methode "Sumathu" handelt. Es hat sich dann herausgestellt, dass diese Methode von Herrn Sulser Max in Thun zusammengestellt worden ist.
Sie verraten uns sicher noch, was Herr Max Sulser in Thun genau macht!

Max Sulser definiert Sumathu als ganzheitliche, energetische Körpertherapie und positioniert sie als "sanfte Alternative" zur Chiropraktik. Der Schwerpunkt der Arbeit liegt im Bereich der Wirbelsäulenund Gelenkbehandlung. Im wesentlichen setzt sich die Methode aus Elementen der klassischen Massage, der Fussreflexzonenmassage und der Akupunkturmassage zusammen.

Wie kann man sich bei soviel Methoden überhaupt einen Überblick verschaffen, welche Methode was bietet?

Das war eine enorme Fleissarbeit für uns; Recherchen im Internet, Rückfragen bei Versicherern, Verbänden und Schulen und Konsultation der Literatur natürlich. Wir haben etwa ein Jahr gebraucht, bis wir von allen Methoden die nötigen umfassenden Unterlagen hatten.

\section{Unter Literatur verstehen Sie wahrscheinlich} den Bücherstand am Bahnhofskiosk?

Nein, es gibt durchaus auch seriöse Literatur zu diesem Thema, aber sie oft schwer zugänglich. Vielfach mussten wir die Informationen bei den Verbänden, Schulen oder sogar den Therapeuten direkt anfordern.

Wieviele Schulen und Verbände der Komplementärmedizin gibt es denn etwa in der Schweiz?

Auch diese Frage können wir erst seit kurzem verbindlich beantworten. Wir haben in unserer Datenbank alle Verbände und Schulen erfasst, die uns von unseren Therapeuten gemeldet worden sind. Diese Angaben waren aber oft veraltet. Von den anfänglich 1000 Datensätzen für Verbände im In- und Ausland blieben nach der minutiösen Kontrolle noch rund 450 übrig, davon 250 in der Schweiz. Bei den Schulen waren es initial etwa 2000, nach der aufwendigen Kontrolle noch rund 1600, davon 900 in der Schweiz. Man muss wissen, dass Schulen und Verbände zum Teil so rasch verschwinden, wie sie gegründet werden. Wir kennen Verbände, die bestehen nur aus zwei Mitgliedern, und Schulen, die nur auf dem Papier existieren. Das muss man aber zuerst herausfinden.

Ihr Anerkennungsprozedere beisst sich doch in den Schwanz: um Therapeuten zu registrieren, fragen Sie nach den Verbänden, und um Verbände zu registrieren, fragen Sie die Therapeuten.

Nicht ganz. Wir haben Schulen, Verbände und auch die Versicherer systematisch befragt und die Angaben der Therapeuten mit Hilfe unserer Datenbank analysiert. Das hat ein sehr gutes erstes Resultat ergeben. Auf diese Art haben wir zum Beispiel erfahren, dass es für die Ohrkerzentherapie keine Ausbildung gibt, die mehr als acht Stunden dauert. Diese Information kam von den Therapeuten, denn einen Verband für Ohrkerzentherapie gibt es nicht. 


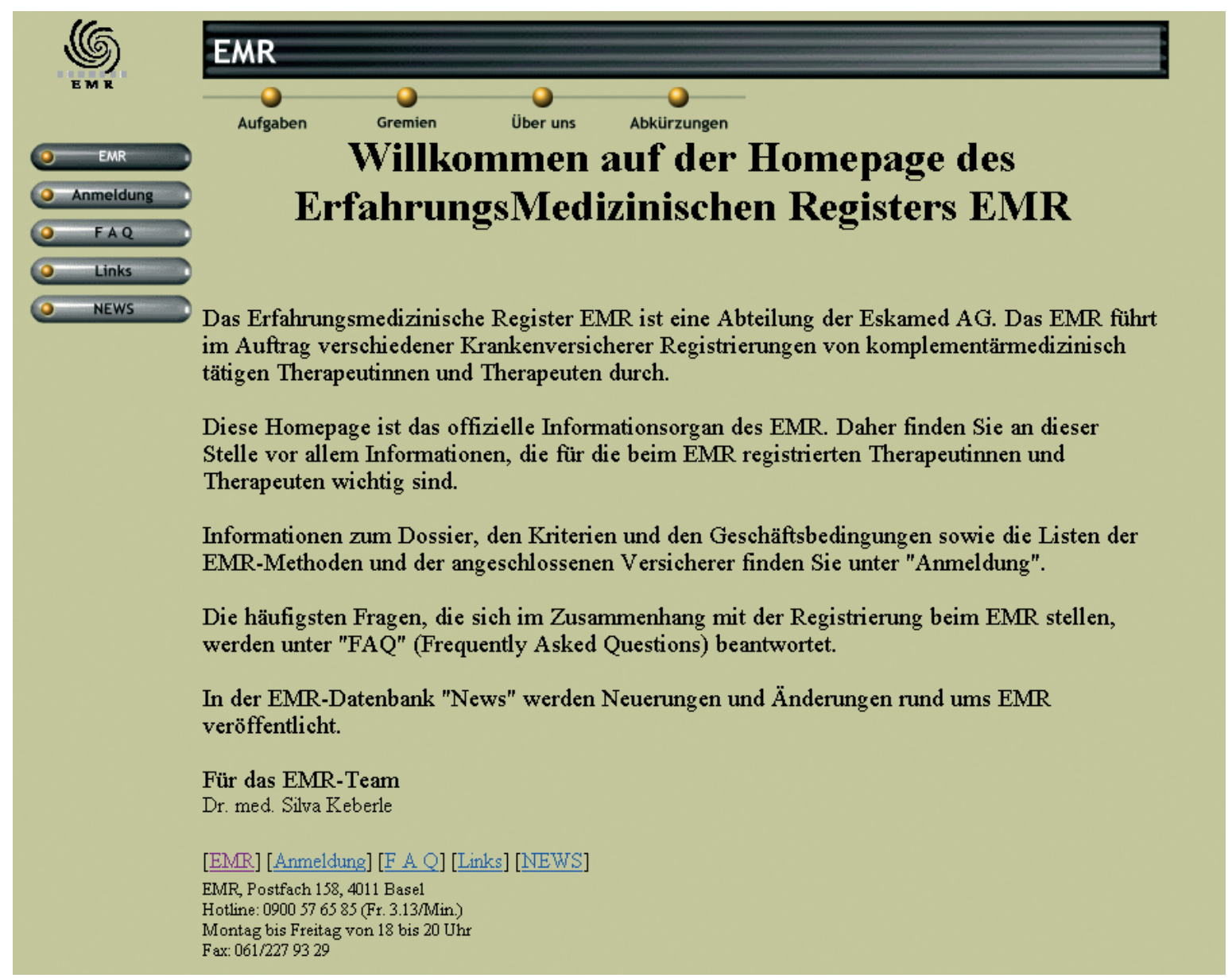

Die Website - das offizielle Organ des EMR

Arbeitet das EMR mit Schulen und Verbänden zusammen?

Ja, natürlich, das war von Anfang an erklärtes Ziel des EMR. Nur muss diese Zusammenarbeit ebenfalls auf einheitlichen Kriterien beruhen und diese mussten basisdemokratisch erarbeitet werden. Dieses Ziel ist bereits erreicht. Zur Zeit wird die Zusammenarbeit mit den qualifizierten Verbänden implementiert, indem wir diesen sogenannten Partnerverbänden die Verantwortung für Fortbildungskontrolle übergeben, auch hier ganz wie bei der FMH. Wir geben also Schritt um Schritt und unter kontrollierten Bedingungen die Verantwortung an die seriösen und entsprechend organisierten Verbände ab.

Warum überlässt man die Qualitätskontrolle nicht ganz den Schulen und Fachverbänden?

Das ist - wie erwähnt - das Ziel, zumindest was die Definition der Qualitätskriterien und die Fortbildungskontrolle betrifft. Das EMR ist auf eine enge Zusammenarbeit mit den guten Fachverbänden und Schulen angewiesen und begrüsst alle Bestrebungen, die dem Ziel der Qualitätssicherung dienen. Es sind ja die Verbände und auch die Schulen, welche am besten die notwendigen Qualitätsstandards für das Erlernen einer Methode der Komplementärmedizin definieren können. Allerdings ist es nicht selten so, dass Verbände oder Schulen, welche dieselben Methoden vertreten, ein rivalisierendes Verhalten untereinander aufweisen, was die emotionslose Erarbeitung objektiver Qualitätskriterien manchmal erschwert. Drum gehen wir schrittweise vor und definieren gemeinsam mit den Betroffenen, unter welchen Bedingungen ein Verband mit dem EMR zusammenarbeiten kann.

Wie gross ist der Aufwand zur Bearbeitung eines Anmeldedossiers im Schnitt?

Ein ganz einfaches Dossier gibt uns Arbeit für mindestens drei Stunden, solche Dossiers sind aber die Minderheit. 70 bis 80 Prozent aller Bewerbungen kommen unvollständig bei uns an, was einen enormen Mehraufwand verursacht. Komplizierte Dossiers, die unvollständig eingereicht werden und bei denen die Unterlagen nicht die benötigten Informationen erhalten, können uns einen bis mehrere Tage beschäftigen, vor allem, wenn sie auch noch von den EMR-Gremien beurteilt werden müssen.

Dem EMR wird vorgeworfen, es arbeite privat und gewinnorientiert. Stimmt das?

Das EMR ist eine Abteilung der Firma Eskamed AG. Die Eskamed gehört mir, ist also privat. Wir arbeiten auch sicher nicht verlustorientiert. Beim EMR war 
aber von Anfang an klar, dass der Aufwand in der ersten Zeit deutlich höher sein würde wie der Ertrag. Wir haben diese Aufgabe sicher nicht wegen des Geldes angenommen, sondern weil das Konzept faszinierend und spannend ist. Das EMR ist aufgrund der enormen Probleme und den dadurch bedingten Folgekosten, die sich immer wieder neu ergeben, bisher finanziell immer noch nicht in einem konsolidierten Bereich. Allein unsere Datenbank hat rund 500 000.- Franken gekostet, sie enthält 2306 Felder, 243 Layouts und 1089 Scripts, und sie muss noch weiter ausgebaut werden.

Wie erfolgt die Qualitätskontrolle bei den Ärztinnen und Ärzten?

Das EMR ist grundsätzlich nur für den Privatversicherungsbereich zuständig. Man muss also unterscheiden zwischen Ärzten, die mit einer oder mehreren der fünf Komplementärmethoden für die Grundversicherung zugelassen sind. Hier gelten ja ausschliesslich die Fähigkeitsausweise der FMH, die von den entsprechenden Gesellschaften der UNION schweizerischer komplementärmedizinischer Ärzteorganisationen verwaltet werden. Alle anderen Ärzte, also zum Beispiel Kollegen aus dem Ausland, die in der Schweiz nie schulmedizinisch gearbeitet haben, können sich beim EMR registrieren lassen. Hier ist allerdings zu sagen, dass die meisten Versicherer in den Bestimmungen ihrer Zusatzversicherung die Ärzte als Leistungserbringer generell akzeptieren, so dass sich eine Registrierung beim EMR erübrigt. Es wäre meiner Meinung nach allerdings sinnvoll, dass für Ärztinnen und Ärzte, die komplementärmedizinisch tätig sind, eine Lösung ohne EMR gefunden wird. Aber das ist Aufgabe der Versicherer und der betroffenen Gesellschaften, das EMR hat hier nicht zu entscheiden. Soviel ich weiss, ist die Sache jedoch auf gutem Weg. Bei den Ärzten, die andere als die in der Grundversorgung aufgeführten Komplementärmethoden praktizieren, prüft das EMR, ob die Bewerber für die Methode, die sie praktizieren, eine Ausbildung absolviert haben. Selbstverständlich betrifft das nur den methodenspezifischen Teil, nicht aber die Schulmedizin. Hier ist die Qualifikation eines Arztes ja unbestritten. Aber wie gesagt, das EMR ist vor allem auf nicht-ärztliche Therapeuten spezialisiert, und ich bin froh, wenn die Versicherer mit den Ärzten hier eine eigene Lösung finden. Ich kann gut verstehen, dass ein Arzt, eine Ärztin irritiert ist, wenn sie im selben Register geführt wird wie die nicht-akadamischen Therapeuten.

Haben Sie bei Registrierungsversuchen auch Druckversuche erlebt? Es geht schliesslich um sehr viel Geld ...

Druckversuche nicht, aber doch einiges an Anfeindungen. Wenn man ein Kontrollsystem einführt, macht man sich selten beliebt, das ist an sich auch verständlich. Schade ist, dass sich einzelne Gruppierungen mit wenig demokratischen Mitteln ihre frühere Position zurückerobern wollen. Aber die Zeiten haben geändert, Qualitätskontrollen gehören zu jedem Berufsbild. Erfreulich ist dafür, dass wir viel positives Echo bekommen und vor allem, dass das EMR viel in Bewegung gebracht hat in Richtung systematische Qualität in der Komplementärmedizin. Und wir sind erst am Anfang dieses Prozesses.

Wer sind eigentlich diese 12000 zugelassenen Therapeuten? Kann man diese Population mit ein paar Kennzahlen versehen? Wieviel davon sind Ärzte?

Wir haben wenig Ärzte auf der Liste, etwa 140. Den Grund habe ich bereits erwähnt. 120 Apotheker haben sich bei uns gemeldet, rund 1800 Krankenschwestern und etwa 900 Physiotherapeuten. 80 Prozent der registrierten Personen sind Frauen, die Männer sind eindeutig in der Minderzahl. Die grosse Mehrheit der Therapeuten sind deutschsprachig, rund 20 Prozent stammen aus der Romandie.

Letzthin ging eine Meldung durch die Medien, die WEKO würde beim EMR eine Abklärung durchführen. Um was geht es?

Die WEKO will abklären, ob das EMR seine marktbeherrschende Stellung missbraucht. So wie man mir gesagt hat, betrifft das vor allem die Frage, ob das EMR den Bewerbern unzulässige Beschränkungen auferlegt, also Bewerber willkürlich vom Markt ausschliesst. Und es betrifft die Frage, ob das EMR bezüglich Gebühren seine marktbeherrschende Stellung missbräuchlich ausnützt. Ich denke, mit meinen Erläuterungen habe ich gezeigt, dass beides nicht der Fall ist. Ein Missbrauch würde dem Interesse des EMR zudem völlig zuwiderlaufen. Dem Interesse, den Therapeutinnen und Therapeuten der Komplementärmedizin eine transparente, einheitliche Qualitätskontrolle zu bieten, zum Nutze der Therapeuten und der Versicherer und zum Schutz der Patienten vor schlecht ausgebildeten Therapeuten.

Sie selbst sind Fachärztin für Innere Medizin FMH und haben sich wie keine andere in diesem Land mit dem Thema Komplementärmedizin befasst. Sie sind nun aufgrund dieser Tätigkeit beim EMR aufgeschlossener gegenüber nicht-schulmedizinischen Angeboten?

Ja, das kann man sicher sagen. Aber ich habe vor allem gelernt, dass Schul- und Komplementärmedizin ihre Stärken und ihre Schwächen haben, und zwar komplementär.

Besten Dank für das Gespräch!

Interview: Markus Trutmann 